\title{
A Traffic-aware Solution for Green Wireless Video Sensor Networks
}

\author{
Filipe Sousa*†, João Dias* ${ }^{*}$, Filipe Ribeiro ${ }^{\ddagger}$, Rui Campos* ${ }^{\ddagger}$, Manuel Ricardo* \\ *Faculdade de Engenharia, Universidade do Porto, Porto, Portugal \\ ${ }^{\dagger}$ Fraunhofer Portugal, Porto, Portugal \\ ${ }^{\ddagger}$ INESC TEC, Porto, Portugal \\ filipe.sousalfraunhofer.pt, \\ ee10126@fe.up.pt, \\ \{filipe.a.ribeiro, rcampos, mricardo\}einesctec.pt
}

\begin{abstract}
The growth of the IP cameras market, due to their low price and high availability, is making Wireless Video Sensor Networks (WVSNs) attractive. In a mesh, multi-hop video surveillance scenario Wi-Fi is the enabling technology for WVSNs, due to its flexibility and low cost. However, WVSNs still suffer from bad performance, throughput unfairness, and energy inefficiency.

Previously, we proposed FM-WiFIX+, a holistic solution to address the problem. FM-WiFIX+ uses FM radio to signal when a video sensor should turn its IEEE 802.11 interface OFF, thus saving energy. Herein, we present a new traffic-aware version of FM-WiFIX+. The results obtained through numerical, simulation, and experimental evaluation demonstrate that the new version can achieve savings in energy consumption up to $84 \%$, while maintaining the levels of performance and throughput fairness.
\end{abstract}

Keywords-Energy-efficiency, FM Radio Data System, Network Performance, Out-of-band Signalling, Wireless Video Sensor Networks.

\section{INTRODUCTION}

Wireless cameras became very popular in the last years due to their low price and easy configuration. A wide range of new applications arisen in the fields of agriculture, military, and security. The Internet Protocol (IP) is used to connect wireless cameras to the Internet, leading to the Internet of Things (IoT) paradigm [1]. The high availability of wireless IP cameras led to their use as video sensors, enabling the acquisition of valuable information and the inference of complex and new contexts [2]. A network formed by distributed video sensors is referred in the literature as a Wireless Video Sensor Network (WVSN). In a WVSN the sensors acquire vast amounts of data in the form of video streams, which are typically transferred to a cloud server, as shown in Fig. 1. IEEE 802.11 is increasingly the wireless technology of choice to do it, since it allows high data rate communications up to hundred meters [3]. For simplifying the WVSN deployment, each node should autoconfigure itself and connect to peer video sensors wirelessly, forming a wireless multi-hop network. However, IEEE 802.11 when applied to multi-hop WVSNs has three problems: poor performance, throughput unfairness, and energy inefficiency [3].

In IEEE 802.11 based multi-hop networks, the Carrier Sense Multiple Access - Collision Avoidance (CSMA/CA) mechanism is not able to efficiently avoid collisions, due to

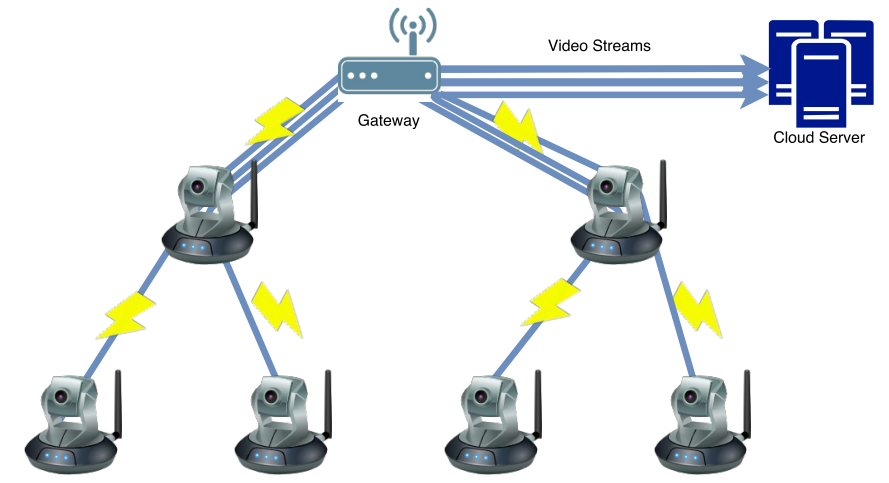

Fig. 1: Video Surveillance scenario.

the presence of hidden nodes. The use of RTS/CTS mechanism may solve the hidden node problem, but in multi-hop networks it introduces the exposed node problem [4]. The hidden nodes and the CSMA/CA backoff mechanism, activated when collisions are detected, leads to poor performance. The throughput unfairness problem is a consequence of the multihop nature of the network, since the medium is monopolised by the nodes nearer the gateway, in detriment of the leaf nodes [5]. Finally, the energy inefficiency is also related to the CSMA/CA mechanism. The network interface is always $\mathrm{ON}$, even when not transmitting or receiving any data. The dominant mode of energy consumption is idle listening, even when the power saving mode is enabled. It can account for $80 \%$ of energy consumption of an IEEE 802.11 station in a busy network and $60 \%$ in a relatively idle network [6].

In [7] a survey on solutions similar to FM-WiFIX+ was conducted. There are many solutions that by using an out-ofband control channel are able to reduce power consumption [8][9] [10][11][12]. New MAC solutions have been proposed to reduce the power consumption in wireless networks [6] and WVSNs [13]. However, there is still margin for improvement, since the Wi-Fi radios can be completely shut down. Energyefficient routing techniques are the most popular approach found in the literature to address the energy inefficiency problem in sensor networks; many solutions are proposed targeting specifically Wi-Fi based WVSNs. Different strategies are employed. The solutions proposed in [14][15] use the information about the energy levels of the network nodes to 
make routing decisions that extend the network lifetime. The absence of solutions to address these problems in a holistic way has motivated the development of the FM-WiFIX concept [3]. The FM-WiFIX concept is built upon the PACE [16] scheduling mechanism, and assumes WiFIX [5] as the routing solution. PACE guarantees equal transmission opportunity for each WVSN node by using in-band signalling, thus assuring throughput fairness. Rather, FM-WiFIX defines the use of out-of-band signalling using low power radios to inform a video sensor when it should transmit and turn its IEEE 802.11 interface OFF to save energy. FM-WiFIX+ is an instantiation of the FM-WiFIX concept. It considers an Frequency Modulated Radio Data System (FM-RDS) based out-of-band control channel and additional mechanisms to enable FM-RDS-based and the collection of topology information.

FM-WiFIX+ was designed for WVSNs operating in saturation mode. Herein, we present a new version that extends the solution to WVSNs operating in non-saturation mode. A traffic-aware mechanism is added to further improve energy efficiency. Each node estimates its offered load and switches OFF its Wi-Fi interface when it knows there is no data to transmit in one or more of the next polling rounds, even if it is instructed by the gateway through the FM-RDS radio to be ON. Also each node notifies the relay nodes in the path to the gateway to switch OFF their Wi-Fi interfaces during the same period of time.

Our main contribution is a new version of FM-WiFIX+, featuring an FM-RDS out-of-band control channel, a scheduler, and a simple traffic-aware mechanism that significantly increases the energy-efficiency of WVSNs in non-saturation mode.

The rest of this paper is organised as follows. Section II describes the FM-WiFIX+ solution, including the registration phase, polling mechanism, the adopted FM-RDS messages format, and the new traffic-aware version of FM-WiFIX+. Section III presents the numerical, simulation, and experimental evaluation performed. Section IV draws the main conclusions and points out the future work.

\section{ThE FM-WIFIX+ SOLUTION}

FM-WiFIX+ [7] is an evolution of PACE to improve the energy efficiency of WVSNs by using FM-RDS out-of-band control channel to switch ON/OFF the Wi-Fi interface of WVSN nodes. In what follows we present the original version of FM-WiFIX+, which was traffic-agnostic. Then, we describe the new traffic-aware version.

\section{A. Traffic-agnostic version}

FM-WiFIX+ includes multiple enhancements to FMWiFIX, in order to achieve an implementable solution. During the network bootstrap, a registration phase starts, so that the gateway is able to collect the WVSN topology. Each node sends a registration message including its parent MAC address. By combining the information through these messages, the gateway learns the active WVSN topology. Then, the gateway sends a registration ACK message per WVSN node in order to assign an 8-bit identifier to each node. The assignment of these IDs avoids the use of MAC addresses, six times greater, and considerably improves the efficiency of the control protocol.
Additionally, the relay nodes seating between a given node and the gateway learn the IDs of their descendants by snooping the registration ACK message sent by the gateway.

After the registration phase, the polling mechanism starts. According to the FM-WiFIX concept, the scheduling mechanism should ensure that each node is polled once every round and the $\mathrm{Wi}-\mathrm{Fi}$ state changes are minimal to decrease the energy consumption of every node. Since the WVSN has a tree topology, a suitable manner of achieving this is by polling the leaf nodes first and then move to the parent nodes. In this way, the leaf nodes can turn their Wi-Fi interfaces OFF as soon the frame arrives at the gateway. The gateway then builds a vector with the polling nodes order.

An FM-RDS message is sent in each polling. The message contains the 8-bit identifier of the node to which the transmission opportunity is granted. When receiving a polling message addressed to one of its children, the relay nodes also turn their Wi-Fi interfaces $\mathrm{ON}$, activating a path from the transmitting node to the gateway. Similarly, when hearing a polling message not addressed either to itself or to one of its children, the node switches its Wi-Fi interface OFF; this avoids the need for control messages to turn Wi-Fi OFF.

Fig. 2 exemplifies the FM-WiFIX+ scheduling mechanism. It illustrates how the nodes switch their Wi-Fi interfaces ON and OFF, according to the control message being broadcast for node $n 4$. The depicted behaviour is repeated for each WVSN node. With this approach, the fairness provided by PACE is kept. The reception of the FM-RDS message by the polled node acts as a trigger for data transmission.

FM-RDS: Polling message for $\mathrm{n} 4$

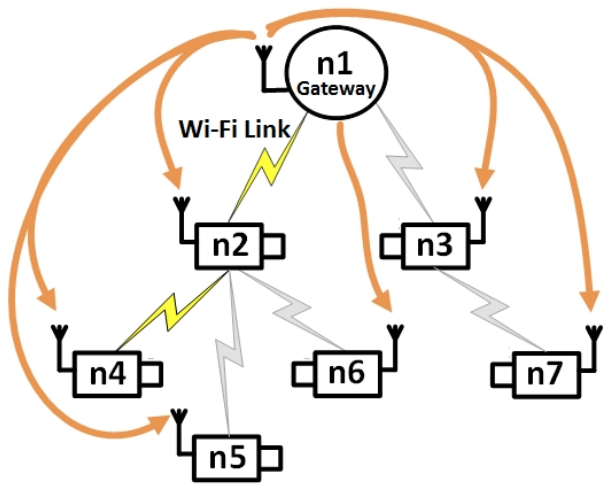

Fig. 2: Upon reception of the FM-RDS polling message to node 4 , only $n 4$ and $n 2$ remain with their Wi-Fi interface ON.

There is, however, a limitation related to the use of the FM-RDS control channel to run the FM-WiFIX+ scheduling mechanism. The standard states that the FM-RDS bitrate must be precisely $1187.5 \mathrm{bit} / \mathrm{s} \pm 0.125 \mathrm{bit} / \mathrm{s}$ [17]. Since the standard also specifies RDS groups of 104 bit and each polling request requires the generation of an entire RDS group, it would take $\frac{104 \mathrm{bit}}{1187.5 \mathrm{bit} / \mathrm{s}} \approx 87.58 \mathrm{~ms}$ to send a polling request. This results in a packet rate of $\approx 11.4$ packets/s, which decreases the maximum throughput of the network to unacceptable levels and makes the control channel the system's bottleneck.

In order to overcome this limitation, the FM-WiFIX+ 
polling mechanism is based on burst transmissions. Instead of transmitting one packet per polling opportunity, a node continuously transmits packets until it receives a new FMRDS message or a timeout occurs. Upon receiving an FM-RDS polling request containing its ID, the video sensor immediately starts its burst transmission. The FM-WiFIX+ polling mechanism is actually better adjusted to the asymmetric traffic pattern of a WVSN; as the sensor nodes are essentially data sources.

The use of FM-RDS [17] as the out-of-band control channel was motivated by the low-cost, low consumption and high range of FM radios. The standard specifies frames of 104 bit, divided in 4 blocks where each block contains an information word (16 bit) and a checkword (10 bit). In our control protocol, 9 bit were used: 8 bit for the node ID and 1 bit for a flag that indicates if a video sensor has data to receive. If the video sensor has data to receive, it turns its Wi-Fi interface $\mathrm{ON}$ and waits for a regular polling request with data from the gateway. For information redundancy purposes the same control information is repeated in the four blocks that form an RDS group, taking advantage of the extra space in each frame.

The purpose of this approach is the reestablishment of the network performance enabled by PACE, despite the limitations of the FM-RDS technology. The generation time of the FMRDS messages is used for burst transmissions, instead of being wasted, thus boosting the WVSN performance. Due to the multi-hop nature of the WVSN the burst must be controlled to avoid the medium is monopolised by the nodes nearer the gateway. During the registration phase a maximum bit rate is computed for each node based on the number hops the node is from the gateway.

\section{B. Traffic-aware version}

FM-WiFIX+ was designed having in mind WVSNs in saturation mode - i.e., each node has always a packet to transmit to the gateway. Herein, we propose a new version targeting WVSN operating in non-saturation mode; namely, the polling mechanism and the corresponding behaviour of the WVSN nodes are modified. This means that we do not have a constant stream during a long period of time. Instead, during a short time a Constant Bit Rate (CBR) video stream is sent to the cloud server; for example, in a video monitoring scenario each video sensor only sends data when movement is detected. In order to avoid nodes having their Wi-Fi radio switched ON, even when they do not have data to transmit, a WVSN node switches OFF its Wi-Fi radio during the period of time it antecipates there will be no traffic to transmit; this is done even if it receives a polling message from the gateway. In each data packet sent to the gateway each node indicates to the relay nodes when the next data packet will be transmitted. For CBR video streams this estimation can be easily performed and may enable further energy savings. Since relay nodes receive information when the next data packet will be transmitted they know when to switch $\mathrm{ON}$ their Wi-Fi radios; the gateway also receives such information and can re-adjust the polling interval. This re-adjustment is only performed after a learning phase in order to capture the traffic pattern associated to each video sensor.

\section{FM-WIFIX+ EVALUATION}

We evaluated the performance and energy consumption of the new FM-WiFIX+ version for the three regular WVSN topologies shown in Fig. 3: 1) binary tree topology; 2) three hop, balanced tree topology; 3) unbalanced tree topology. In what follows, we present the numerical, simulation, and experimental evaluation. Each simulation and experiment was repeated multiple times, in order to obtain statistically relevant results and narrow $95 \%$ confidence intervals. The FM-WiFIX+ energy-efficiency gain and performance are compared to the ones achieved by PACE and legacy CSMA/CA when they are used in the same context. PACE was designed to improve the WVSN performance and fairness; the corresponding evaluation results were presented in [16]. In this section we evaluate if FM-WiFIX+ achieves the same performance and fairness results, and calculate its energy gains.

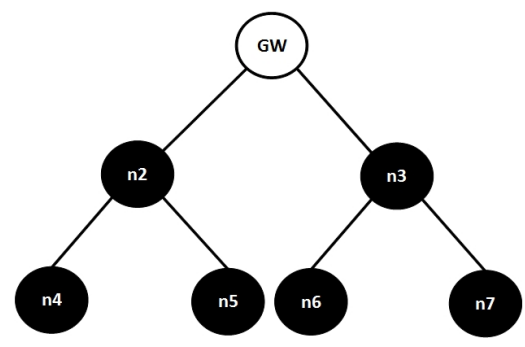

(a) Binary Tree Topology.

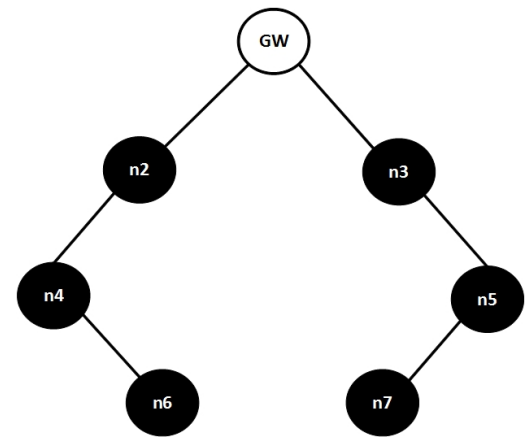

(b) A balanced tree topology with two branches and three hops.

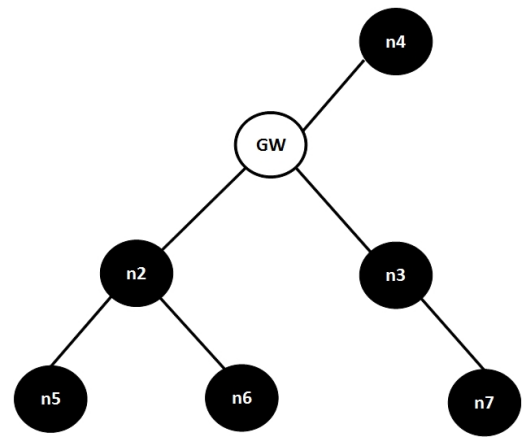

(c) Unbalanced tree topology.

Fig. 3: The three regular WVSN topologies used to evaluate the new version of FM-WiFIX+. 


\section{A. Numerical Analysis}

We assume that all nodes in the WVSN transmit a video stream to the gateway. Since the energy is calculated by multiplying the necessary power to transmit/receive a packet by the amount of time that is necessary do it as before we need to find the amount of time spent in transmission, receiving, and idle modes. Since for PACE and CSMA/CA all nodes are active even when not transmitting/receiving packets, the equation simply needs to consider the power of each Wi-Fi interface $P_{W i-F i}$, multiplied by the total duration of the network operation $T_{o p}$. For obtaining the total energy consumed by the WVSN we multiply this by the number of nodes, $N$ (excluding the gateway). (1) presents the total amount of energy that is necessary for each node of WVSN to transmit a video stream to the gateway, when using PACE.

$$
E_{P A C E}=P_{W i-F i} \cdot T_{o p} \cdot N
$$

In FM-WiFIX+ the Wi-Fi interface of each WVSN node is turned OFF whenever the node is not transmitting. This requires the calculation of the time that each node is actively transmitting the video stream to the gateway, and thus spending energy. In [3] we already presented a numerical analysis for the binary topology. The previous equation was reformulated to be used for the three regular topologies shown in Fig. 3. The first term of (2) is a sum from 1 to $h_{k}$, the number of hops of branch $k$. $L$ represents the total number of branches for a topology. In the sum, $i^{2}$ represents the total number of time spent by nodes to transmit a frame to the gateway. This term is multiplied by $T$, the time required for a node to transmit a packet to its neighbor and receive ACK packet. The second term $T_{c o r}$ is used to cancel the transmissions of each relay node already considered in the first term. The sums are multiplied by $M$ which represents the average number of messages sent by each node to the gateway during the operation time of the WVSN.

$$
t_{F M-W i F I X+}=\left(\sum_{k=1}^{L}\left(\sum_{i=1}^{h_{k}} i^{2} \cdot T\right)-T_{c o r}\right) \cdot M
$$

The total network energy is calculated using (3). In the first term the total network time is multiplied by the Wi-Fi idle power $P_{\text {idle }}$.

$$
E_{F M-W i F I X+}=P_{i d l e} \cdot t_{F M-W i F I X+}+P_{F M} \cdot T_{o p} \cdot N
$$

The power spent with the FM-RDS radio transceiver $P_{F M}$ is included in the second term and multiplied by the network operation $T_{o p}$ and by the number of nodes, $N$ that have the FM-RDS radio transceiver. Moreover, only the idle power of the Wi-Fi interface is considered since Wi-Fi idle listening and receive are the dominant modes of energy consumption [6].

The energy savings can be calculated using the following equation:

$$
E_{\text {savings }}=1-\frac{t_{F M-W i F I X+}}{t_{P A C E}}
$$

\section{B. Simulation Setup}

FM-WiFIX+ was implemented in network simulator 3 (ns-3 version 3.24.1) including 1) the topology information protocol, which is used to calculate the polling vector; 2) the scheduling mechanism with a burst transmission for each polling message; 3) traffic-aware mechanism; 4) emulation of the FM-RDS channel. To overcome the FM-RDS low bit rate limitation, the FM-WiFIX+ polling mechanism was implemented based on burst transmissions; instead of transmitting one packet per polling opportunity, a node continuously transmits packets until it receives a new control message or a timeout occurs. Moreover a traffic-aware mechanism was implemented to further improve the energy consumption of the WVSN. Finally, to emulate the FM-RDS channel a second Wi-Fi interface was used and modified to generate a FM-RDS polling message each $154 \mathrm{~ms}$. This value was also used in the experimental setup and is the result of experimental tests.

In all simulations, the size of data frames was set to 1200 bytes. The full set of simulation parameters is summarized in Table I.

TABLE I: Simulation parameters

\begin{tabular}{|c|c|}
\hline Simulation Variable & Value \\
\hline No. runs per test & 30 \\
\hline Distance & $80 \mathrm{~m}$ \\
\hline RxGain & $5 \mathrm{~dB}$ \\
\hline TxPowerStart & $16 \mathrm{~dB}$ \\
\hline TxPowerEnd & $16 \mathrm{~dB}$ \\
\hline Fragmentation & Disabled \\
\hline Packet Size & 1200 bytes \\
\hline RTS/CTS & Disabled \\
\hline Mobility model & None \\
\hline Error ratio model & Nist Error Ratio Model \\
\hline Communication standard & IEEE $802.11 \mathrm{~b}$ \\
\hline Data rate & $11 \mathrm{Mbps}$ \\
\hline
\end{tabular}

\section{Experimental Setup}

In order to deploy a low cost WVSN prototype, the hardware chosen was the Raspberry Pi Model B, Revision 2.0 platform, along with the Wi-Fi dongle TP-LINK TL-WN823N and the Raspberry Pi's camera module. For generating the FM RDS broadcast at the gateway, an open-source program turns the Raspberry Pi into an FM transmitter using its built-in PulseWidth Modulation (PWM) generator. On the sensor side, the SparkFun FM Tuner Evaluation Board - Si4703 was used to receive the FM RDS broadcast, together with a software for controlling the FM tuner with the Raspberry Pi. Antennas were used at the transmitter and receivers, in order to ensure the RDS reception.

The assembled testbed was composed by a gateway and six video sensor nodes (see Fig. 3a), operating in Wi-Fi ad-hoc mode. The goal of the experiments was to evaluate the performance and energy consumption of FM-WiFIX+ solution for a binary tree topology. Afterwards, its gains and performance were compared to the ones achieved by PACE in the same scenario. The Iperf tool was used to generate traffic in every node at a constant bit rate of $350 \mathrm{kbit} / \mathrm{s}$, which was enough to saturate the network and provide statistics about the network performance. The experiments consisted in generating traffic to the gateway simultaneously from all video sensors. Each 
sensor sent its data during $60 \mathrm{~s}$. At the end of each experiment, the Iperf server calculated the statistics for each sensor and saved them in a log file. Each experiment was repeated 8 times for the three topologies shown in Fig. 3. Using the Student's t-distribution, due to the small sizes of the data sets, $95 \%$ confidence intervals were calculated.

\section{Results}

FM-WiFIX+ is now evaluated based on the results obtained from numerical analysis, simulation and experimental tests. For the three regular topologies Fig. 3, FM-WiFIX+ was compared against PACE and legacy CSMA/CA.

Fig. 4 shows the throughput results obtained for FMWiFIX+ and PACE. FM-WiFIX+ always achieves a slightly better throughput than PACE. For the burst mechanism of FM-WiFIX+ the scheduler assigns equal time slots to all nodes. As a consequence, nodes closer to the gateway can transmit more information than leaf nodes. In PACE, every node receives exactly one polling opportunity per round, and all nodes are given the opportunity to send one packet. FM$\mathrm{WiFIX}+$ guarantees equal access to the medium to every node by assigning the same time slot size to every node; yet, the nodes closer to the gateway can transmit more packets in the same slot than the leaf nodes. By using this strategy FMWiFIX+ enables higher aggregated network throughput. The throughput values obtained are low due to limitations found [18].

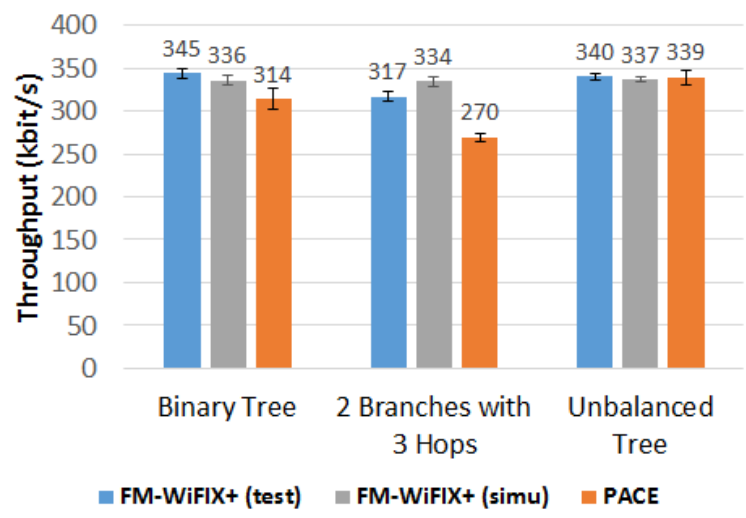

Fig. 4: Throughput achieved by FM-WiFIX+ and PACE during testbed experiments and simulations for the three scenarios.

Due to the experimental limitations we performed a numerical analysis and simulations for different offered loads. We evaluated the impact of different offered loads in the total energy savings. These values are calculated as the ratio between the total network energy spent for FM-WiFIX+ (3) and the total network energy consumed by PACE (1). $E_{F M-W i F I X+}$ is calculated by multiplying the time that each node was ON by the Wi-Fi dongle power consumption in the idle state and adding the power of all FM radios multiplied by the network operation time. $E_{P A C E}$ is calculated by multiplying the total number of nodes by the Wi-Fi power consumption and by network operation time.

The results in Fig. 5a show that the traffic-aware version of FM-WiFIX+ can achieve energy savings up to $84 \%$ for a binary tree topology; for the other two regular topologies the energy savings are even greater and can be easily computed using the equations presented in Section III-A. For low offered loads since the nodes do not need to transmit data so often, Wi-Fi interfaces can be OFF, thus saving more energy. For offered loads higher than $3.1 \mathrm{Mbit} / \mathrm{s}$ the energy savings are $65 \%$. FM-WiFIX+ includes a traffic shaper to control the burst, so that the medium is not monopolised by the nodes nearer the gateway. This traffic shaper is responsible for stabilizing the energy savings. As expected, the energy savings for the traffic-agnostic FM-WiFIX+ version are constant (cf. Fig. 5a); this value was also achieved during the experimental tests.

Fig. 5b presents the overall performance achieve by FMWiFIX+ and CSMA/CA. As expected FM-WiFIX+ network throughput is constant when saturation is reached; also, the saturation point is reached for offered loads greater than those obtained when CSMA/CA is used. Since in FM-WiFIX+ each node has a time slot to transmit information, the Jain's index, which measures the level of fairness, is constant. CSMA/CA fairness decreases when network throughput capacity reaches the limit, meaning that nodes closer to the gateway will gain more access to the medium than the rest. FM-WiFIX+ not only can achieve energy savings up to $84 \%$ but also keep the same performance and fairness for higher offered network loads.

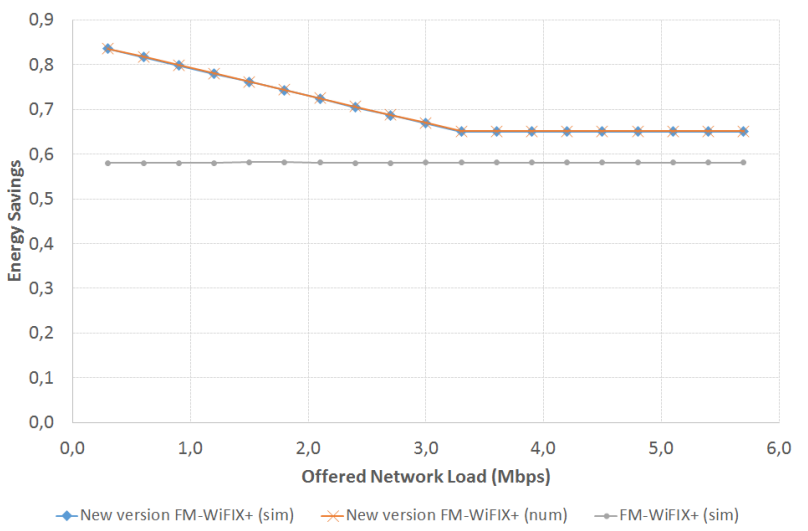

(a) Energy savings for different offered network loads.

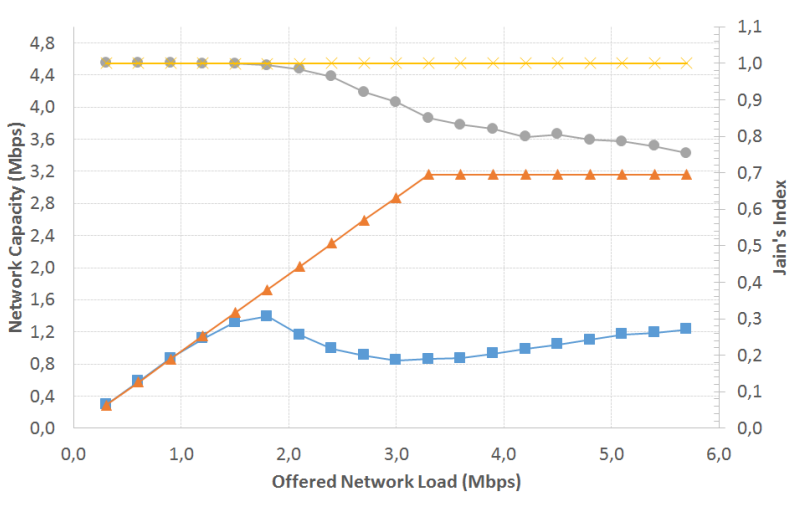

-CSMA Net. Capacity - A-FM-WiFIX+ Net. Capacity - - CSMA Jain's Index - FM-WiFIX+ Jain's Index

(b) FM-WiFIX+ performance for different offered loads.

Fig. 5: Impact in energy savings and performance of FMWiFIX+ for different offered network loads. 


\section{CONCLUSions}

We evaluated the traffic-aware FM-WiFIX+ version and showed that it is a solution addressing the three problems of WVSN: bad performance, throughput unfairness, and energy inefficiency. The performed analysis was conducted through numerical, simulation, and experimental evaluation. The new FM-WiFIX+ version enables significant energy savings, reaching $84 \%$ for low offered loads while keeping the performance achieved by PACE.

As future work we will consider the adoption of other radio technologies for out-of-band signalling. Moreover, we will conduct simulations with random topologies and higher network sizes, and generalize our numerical analysis to any WVSN topology. We will also consider the frame aggregation in relay nodes and spatial reuse for further improving WVSN performance and energy-efficiency.

\section{REFERENCES}

[1] L. Mainetti, L. Patrono, and A. Vilei, "Evolution of wireless sensor networks towards the internet of things: A survey," in Software, Telecommunications and Computer Networks (SoftCOM), 2011 19th International Conference on. IEEE, 2011, pp. 1-6.

[2] S.-Y. Chien, T.-Y. Cheng, S.-H. Ou, C.-C. Chiu, C.-H. Lee, V. S. Somayazulu, and Y.-K. Chen, "Power consumption analysis for distributed video sensors in machine-to-machine networks," Emerging and Selected Topics in Circuits and Systems, IEEE Journal on, vol. 3, no. 1, pp. 5564, 2013

[3] F. Sousa, R. Campos, and M. Ricardo, "Energy-efficient wireless multimedia sensor networks using FM as a control channel," in Computers and Communication (ISCC), 2014 IEEE Symposium on, June 2014, pp. $1-7$.

[4] A. Jayasuriya, S. Perreau, A. Dadej, and S. Gordon, "Hidden vs exposed terminal problem in ad hoc networks," Ph.D. dissertation, ATNAC 2004, 2004.

[5] R. Campos, R. Duarte, F. Sousa, M. Ricardo, and J. Ruela, "Network infrastructure extension using 802.1D-based wireless mesh networks," Wireless Communications and Mobile Computing, vol. 11, no. 1, pp 67-89, 2011. [Online]. Available: http://dx.doi.org/10.1002/wcm.916

[6] X. Zhang and K. G. Shin, "E-mili: energy-minimizing idle listening in wireless networks," Mobile Computing, IEEE Transactions on, vol. 11, no. 9, pp. 1441-1454, 2012

[7] J. Dias, F. Sousa, F. Ribeiro, R. Campos, and M. Ricardo, "Green wireless video sensor networks using FM radio system as control channel," in 2016 12th Annual Conference on Wireless On-demand Network Systems and Services (WONS). IEEE, 2016, pp. 1-8.

[8] J. L. da Silva Jr, J. Shamberger, M. J. Ammer, C. Guo, S. Li, R. Shah, T. Tuan, M. Sheets, J. M. Rabaey, B. Nikolic et al., "Design methodology for picoradio networks," in Design, Automation and Test in Europe, 2001. Conference and Exhibition 2001. Proceedings. IEEE, 2001, pp. 314-323.

[9] A. Rowe, R. Mangharam, and R. Rajkumar, "Firefly: A time synchronized real-time sensor networking platform," Wireless Ad Hoc Networking: Personal-Area, Local-Area, and the Sensory-Area Networks, CRC Press Book, 2006.

[10] B. Van der Doorn, W. Kavelaars, and K. Langendoen, "A prototype low-cost wakeup radio for the $868 \mathrm{mhz}$ band," International Journal of Sensor Networks, vol. 5, no. 1, pp. 22-32, 2009.

[11] E. Shih, P. Bahl, and M. J. Sinclair, "Wake on wireless: an event driven energy saving strategy for battery operated devices," in Proceedings of the 8th annual international conference on Mobile computing and networking. ACM, 2002, pp. 160-171.

[12] I. Haratcherev, M. Fiorito, and C. Balageas, "Low-power sleep mode and out-of-band wake-up for indoor access points," in GLOBECOM Workshops, 2009 IEEE. IEEE, 2009, pp. 1-6.
[13] Q. Zhou, Y. Xu, and X. Li, "HTSMAC: High throughput sensor MAC for wireless video networks," in Wireless Communications, Networking and Mobile Computing, 2007. WiCom 2007. International Conference on. IEEE, 2007, pp. 2428-2431.

[14] M. Chen, V. Leung, S. Mao, and Y. Yuan, "Directional geographical routing for real-time video communications in wireless sensor networks," Computer Communications, vol. 30, no. 17, pp. 3368-3383, 2007.

[15] I. Politis, M. Tsagkaropoulos, T. Dagiuklas, and S. Kotsopoulos, "Power efficient video multipath transmission over wireless multimedia sensor networks," Mobile Networks and Applications, vol. 13, no. 3-4, pp. 274-284, 2008.

[16] F. Ribeiro, R. Campos, D. Rua, C. Pinho, and J. Ruela, "PACE: Simple multi-hop scheduling for single-radio 802.11-based stub wireless mesh networks," in Wireless and Mobile Computing, Networking and Communications (WiMob), 2013 IEEE 9th International Conference on, Oct 2013, pp. 103-110.

[17] N. Européenne, "Specification of the radio data system (RDS) for VHF/FM sound broadcasting in the frequency range from 87,5 to 108,0 MHz," April 1998.

[18] J. Dias, "Mesh network of surveillance cameras using fm radio as a control channel," Master's thesis, Faculdade de Engenharia da Universidade do Porto, 2015, [Accessed: Aug. 22, 2015]. [Online]. Available: http://paginas.fe.up.pt//ee10126/Tese/wpcontent/uploads/2015/08/JoaoDias201000634Thesis.pdf 